\title{
L'habit fait-il le moine ? Quelques réflexions autour des proverbes vestimentaires du Moyen Âge
}

\section{Fanny Oudin}

\section{(2) OpenEdition}

1 Journals

Édition électronique

URL : http://journals.openedition.org/questes/102

DOI : 10.4000 /questes. 102

ISSN : 2109-9472

Éditeur

Les Amis de Questes

\section{Édition imprimée}

Date de publication : 15 avril 2013

Pagination : 1-22

ISSN : 2102-7188

\section{Référence électronique}

Fanny Oudin, «L'habit fait-il le moine? Quelques réflexions autour des proverbes vestimentaires du Moyen Âge », Questes [En ligne], 25 | 2013, mis en ligne le 01 janvier 2014, consulté le 22 septembre 2020. URL : http://journals.openedition.org/questes/102 ; DOI : https://doi.org/10.4000/questes.102

\section{(C) Association des amis de «Questes »}




\title{
L'habit fait-il le moine? Quelques réflexions autour des proverbes vestimentaires du Moyen Âge
}

\author{
Fanny OUDIN \\ Université Paris-Sorbonne (Paris IV)
}

«L'habit ne fait pas le moine ». Lapidaire, le proverbe ne s'attarde pas sur la matérialité du vêtement, dont il fait l'économie ${ }^{1}$. Il pose d'emblée une équivalence entre le vêtement, conçu comme un tout, et une catégorie sociale. Cette équivalence, il la nie, tandis que d'autres proverbes, comme «drap font et deffont la gent », ou sa variante « robe refait mont home $»^{2}$, la posent comme acquise ... non sans jeter, par le biais de la dérivation entre «faire », « deffaire » et "refaire », un regard suspicieux sur l'opération dénotée par l'équivoque verbe «faire », qui reste entachée, même lorsqu'elle paraît réussie, d'une instabilité de mauvais aloi. Qu'il fasse ou non le moine, le vêtement ne parait guère fiable. Les proverbes ont des allures satiriques, mais sans que l'objet visé n'apparaisse clairement. Faut-il, en effet, y voir une mise en garde contre les séductions du vêtement, et comprendre que l'habit ne fait pas le moine, ni ne le doit ? Ou bien qu'il fait le moine mais ne le devrait pas? Faut-il, au contraire, considérer que le proverbe déplore l'entorse faite par la réalité des pratiques à une situation idéale où le moine et son habit seraient en parfaite adéquation, et entendre que l'habit ne fait pas le moine, mais qu'il le devrait, ou qu'il le fait, mais pas comme il le devrait? Et que signifie exactement, au juste, «faire le moine »? La capacité de ce semi-auxiliaire

\footnotetext{
${ }^{1}$ Nous la ferons aussi, et renverrons, pour tout ce qui concerne l'histoire des objets matériels, à la première section de la très sélective bibliographie qui clôt ce volume.

2 Joseph MoRAWski, Proverbes français antérieurs au $X V^{e}$ siècle, Paris, Champion, CFMA, 1925, proverbe $\mathrm{n}^{\circ} 2220$.
} 
à remplacer d'autres verbes ouvre plusieurs possibilités. « Faire le moine », ce peut être, assez banalement, le faire connaître, le désigner, le dire, le signifier - quoique, si l'on en croit un autre proverbe, «l'en ne connoist pas les genz aus drapiaus». "Faire le moine», ce peut aussi être le contrefaire, le jouer - et là encore, l'habit se retrouve instrumentalisé par une mystification, contre laquelle les proverbes mettraient en garde : l'habit ne ferait pas le moine, parce qu'il n'en serait que l'écorce vide. S'ouvre alors une troisième possibilité, où faire conserverait un sens plein, et suggérerait une performativité du vêtement, dont la dimension pragmatique, prise au sens fort, ne consisterait pas seulement à protéger efficacement contre les intempéries, mais établirait une corrélation, une interdépendance entre la modification de l'apparence et la transformation de l'essence. Qu'on lui nie son énigmatique capacité à agir ou qu'on la lui reconnaisse, l'habit, manifestement, ne se contente donc pas de recouvrir le corps, mais, ce faisant, accomplit quelque geste supplémentaire, dont la nature reste à déterminer, pour mieux faire résonner le doute exprimé par les proverbes, et lui donner sa juste portée. Vouloir, de proverbes aussi ambigus que contradictoires, faire la trame d'une réflexion sur les usages et la conception du vêtement au Moyen Âge peut, certes, paraître une vaine gageure. Mais leurs divergences mêmes ne sont pas sans faire apparaître des convergences qui, tel un fil d'Ariane, nous aideront à déterminer quel crédit les hommes du Moyen Âge purent accorder aux apparences vestimentaires dans la représentation de la personne au sein de la société. 
«Dire» et «connaître" le moine à travers son habit : le système des signes vestimentaires

\section{«Materiam verbis veniat vestire poesis ${ }^{3}$ : le vêtement et le langage}

Postuler une équivalence entre le moine et son habit revient à considérer le vêtement non seulement comme un objet matériel, mais comme un signe, c'est-à-dire un élément qui ne vaut pas uniquement en lui-même et pour lui-même, mais renvoie à autre chose. Ce renvoi, en principe transparent, à son signifié, le signe l'opère non de manière isolée, mais dans le cadre d'un système différentiel qui, par le jeu de normes collectives, lui confère sa valeur. Ainsi, l'habit peut dire un statut social, comme, dans le cas du moine, l'appartenance à un ordre régulier et le choix d'une vie menée hors du «siecle». Les particularités de ce vêtement pourront, à leur tour, préciser l'ordre dont il s'agit : les franciscains sont des cordeliers, et parmi les bénédictins, les clunisiens des moines noirs, les cisterciens des moines blancs. Seul le système des ordres monastiques médiévaux donne, arbitrairement, leur valeur à ces couleurs, une valeur qui sera donc dite signifiante, plutôt que symbolique. Cette valeur, le vêtement l'acquiert par le jeu d'une fonctionnalisation sociale : il devient un signe lorsque la société impose des règles à sa fabrication et à son utilisation au sein d'un système formel normatif et organisé ${ }^{4}$. Que, dans le proverbe, cette dynamique se soit cristallisée autour du moine, n'est donc pas anodin : ne se définit-il pas, plus explicitement qu'un autre, par l'application de règles, comme l'indique l'expression même d'ordre régulier? Parmi tous les états dont se compose une société, il est celui qui met le plus en exergue la

\footnotetext{
3 "Que la poésie vienne vêtir la matière de mots », Geoffroi DE VINSAUF, Poetria Nova, v. 61, cité par Edmond FARAL, Les arts poétiques du XII et du XIII siècle, Paris, Champion, «Bibliothèque de l'école des hautes études », 1962, p. 199 (1 ${ }^{\text {ère }}$ éd. 1924).

4 Roland BARTHES, «Histoire et sociologie du vêtement: Quelques observations méthodologiques », Annales. Histoire, Sciences sociales, $12^{\mathrm{e}}$ année, $\mathrm{n}^{\circ} 3$ (1957), p. $430-441$.
} 
codification et la règlementation qui sont à la base de la valeur signifiante du vêtement. L'habit fait ainsi le moine au sens où le signe suppose une identité entre un vêtement et un statut social: le vêtement, par ses différentes composantes, s'identifie à la position sociale de son porteur, qui se trouve identifié par lui. Dans cette perspective, le jugement exprimé par le proverbe est invalidé, ou du moins, on peut penser que le proverbe dénonce un brouillage de cette identification par le déguisement: normalement, «Len [connoist] les gens aus drapiaus », à défaut de pouvoir les juger par ce biais ${ }^{5}$.

Les vêtements formeraient donc, au sein d'une société donnée, un code sémiotique dont le fonctionnement serait analogue à celui de la langue, et pourrait se penser sur le même modèle ${ }^{6}$. Dans une conception élargie de la communication, le vêtement devient l'un des canaux de la communication non-verbale, au même titre que le geste. Dans l'argumentation sous-jacente à tout discours, il participe de la preuve éthique, qui repose sur l'ethos, l'identité sociale et morale du locuteur, qui rend sa parole fiable et acceptable. Cette participation à la communication, et à la définition de l'ethos de l'orateur explique, entre autres, l'importance fondamentale du costume sur la scène de théâtre. Or, de cette parenté entre le vêtement et le langage, le Moyen Âge semble avoir eu une conscience aiguë, comme en témoigne l'utilisation de la métaphore du vêtement dans plusieurs des arts poétiques édités par Edmond Faral $^{7}$. Toutefois, entre

\footnotetext{
${ }^{5}$ Joseph MoRAWski, Proverbes français..., op. cit., proverbe $\mathrm{n}^{\circ} 1481$, et sa variante « On ne doit pas jugier d'un homme par son habit ».

${ }^{6}$ Cette transparence identitaire entre le signifiant vestimentaire et son signifié est, bien sûr, au fondement d'une figure comme l'allégorie, qui postule une équivalence stricte entre l'être de la personnification allégorique, signifié par son nom, son apparence et son comportement ou ses discours.

7 Edmond FARAL, Les arts poétiques, op. cit. Sur l'importance de la métaphore vestimentaire dans la pensée rhétorique du Moyen Âge, voir en particulier l'article de Mary Frances BROWN, "Critique and complicity: metapoetical reflections on the gendered figures of body and text in the Roman de la rose », Exemplaria, 21:2 (2009), p. $129-159$.
} 
l'utilisation médiévale du vêtement dans le cadre d'une théorie de l'ornement poétique et l'emploi par Roland Barthes du système linguistique comme modèle $\mathrm{du}$ fonctionnement sémiotique du vêtement, un renversement de perspective se fait jour. Alors que, pour nous, le langage sert à penser par métaphore le vêtement, pour le Moyen Âge, c'est le vêtement, qui, par métaphore, sert à penser le langage. Cette différence d'orientation est peut-être liée au fait que Barthes cherchait à mettre en valeur la dimension sémiotique du vêtement, sa «transparence » par-delà son épaisseur matérielle d'objet, tandis que le Moyen Âge viserait, par cette métaphore, à faire sentir l'épaisseur du langage à travers le vêtement.

\section{«L'habit ne fait pas le moine, ne le cuevrechief la béguine»: costume, parure et ornement}

De fait, alors que Barthes met plutôt l'accent sur la langue vestimentaire comme fonctionnalisation générale et codifiée, le Moyen Âge, lui, est plus sensible à l'ornement linguistique et vestimentaire, le surplus du style ou de la parure. Cette orientation est sensible dans sa tendance à procéder par métonymie dans les proverbes : l'«habit» peut y être remplacé par la «gonne» ou la «chape», et complété, pour la nonne, par le «cuevrechief $»^{8}$. Elle se retrouve jusque dans la conception de ce qui réunit vêtement et langage, et fonde la métaphore. Le Moyen Âge en effet, plus que la linguistique moderne, est sensible au fait que, s'il est possible de penser le vêtement par le biais du langage et réciproquement, c'est en vertu de leur commune participation à la nature humaine dans ce qui la différencie de la nature animale. Le

\footnotetext{
${ }^{8}$ Joseph Morawski donne deux variantes du proverbe : « la chape ne fait pas le moine » et "l'habit ne fait pas le moine ne le cuevrechief la béguine" (Joseph MoRAWSKI, Proverbes français..., op. cit., proverbe $\mathrm{n}^{\circ}$ 1053). On trouve dans le DMF une troisième variante, « la gonne ne fait point le moine » (Hiltrud GERNER, article «gonne », dans Robert MARTIN et Bernard COMBETTES (dir.), Dictionnaire du Moyen Français (1330-1500), ATILF, Nancy, 2010, http://www.atilf.fr/dmf).
} 
vêtement, comme le langage, fait partie des manifestations de la rationalité, qui définit l'humain, et fait de lui un être de culture. L'habit et le langage font l'homme en somme, en lui permettant de se différencier des autres vivants. La « beste », elle, est «mue » (muette) et « nue $»{ }^{9}$. Il est donc logique que l'un puisse servir à penser l'autre. Or il est frappant de voir que, chez Albert le Grand par exemple, plus que l'emploi du langage ou du vêtement en soi, c'est l'ornement qui permet de distinguer l'homme, et de dire si telle espèce est humaine ou non. N'est pas humain celui qui « ne fait usage, dans ses discours, ni des procédés rhétoriques, ni des procédés poétiques $\gg{ }^{10}$. De ce point de vue, le choix des arts poétiques, qui utilisent plutôt la métaphore architecturale pour évoquer l'inventio et la métaphore vestimentaire pour l'elocutio, est moins anodin qu'il n'y parait ${ }^{11}$ : la dimension ornementale du vêtement s'en trouve valorisée.

Reste à définir l'ornement lui-même, ce qui conduit à la distinction entre costume et parure $^{12}$. La gratuité de certains éléments du vêtement serait double, et vaudrait à la fois par rapport à leur fonction de protection, et par rapport à leur fonction de signification. N'étant ni utiles d'un point de vue pratique, ni fonctionnalisés, intégrés par le code vestimentaire, les « ornements », la « parure », correspondraient à tout ce qui ne définit pas une identité sociale, mais a plutôt une valeur esthétique, liée à la beauté et

${ }^{9}$ Il suffit, à cet égard, de se rappeler que, dans les romans, la perte de la raison entraîne la transformation en homme sauvage, laquelle a pour première manifestation la perte des vêtements.

${ }^{10}$ Albertus Magnus, De animalibus libri XXVI, Hermann Stadler (éd.), Münster, Aschendorffsche Verlagsbuchhandlung, « Beiträge zur Geischichte der Philosophie des Mittelalters », 2 vol., 1920-1916, t. 2, XXI. 1. 2. 13 : "propter quod etiam rhetoricis persuasionibus in loquendo non utitur neque poeticis $»$.

${ }^{11}$ Geoffroi DE Vinsauf, par exemple, dans le prologue de sa Poetria nova, introduit l'inventio materiae intellectivae (l'inventio proprement dite), par les mots «si quis habet fundare domum ", "si quelqu'un doit bâtir une maison » et l'inventio materiae sensitivae (l'elocutio), par les mots « materiam verbis veniat vestire poesis », "Que la poésie vienne vêtir la matière de mots » (Geoffroi DE VINSAUF, Poetria nova, cité par Edmond FARAL, Les arts poétiques, op. cit., v. 43 et 61).

${ }^{12}$ Roland BARTHES la reprend dans son article « Histoire et sociologie du vêtement », art. cit., p. 433-434. 
au plaisir des sens ${ }^{13}$. Serait ornemental ce qui implique, de la part du sujet, une plus grande liberté d'utilisation, donc une plus grande gratuité, la « parure » intégrant ainsi l'opposition du nécessaire et du superflu. Il entre cependant dans cette affirmation une erreur de perspective, que révèle, justement, le sémantisme de parer. Ce verbe, en ancien français signifie « apprêter, préparer », et implique ainsi de pourvoir au nécessaire, avant même de renvoyer à l'idée d'ornement ${ }^{14}$. La parure implique alors à la fois la fonctionnalisation, au moins matérielle, et le surplus par rapport aux valeurs sociales et au rôle de protection du vêtement, ce qui paraît paradoxal. En réalité, l'explication tient à l'opposition qui existe, en linguistique, entre la langue et la parole, la première renvoyant, comme le rappelle Roland Barthes, à l'institution sociale, au code, la seconde à la « manifestation actualisée de la fonction de langage » qu'il considère, dans le cas du vêtement, comme «faiblement significati[ve]» ${ }^{15}$. Barthes, qui suit Saussure, s'intéresse plus au système sémiotique pour lui-même qu'à la dimension pragmatique du langage vestimentaire, liée à la mise en œuvre par le locuteur: la parole l'intéresse moins que la langue. Pourtant, l'accumulation des pièces vestimentaires ne suffit pas à bâtir une apparence, qui est toujours le fruit d'une élaboration personnelle: la

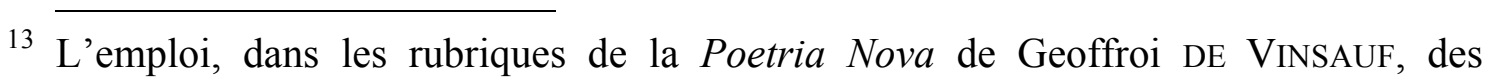
expressions inventio materiae intellectivae et inventio materiae sensitivae pour l'inventio et l'elocutio peut paraître, à cet égard, révélatrive (Geoffroi DE VINSAUF, Poetria nova, cité par Edmond FARAL, Les arts poétiques, op. cit., p. 198-199).

${ }^{14}$ Voir les différents sens de parer dans le Dictionnaire de l'ancienne langue française de Frédéric Godefroy (Dictionnaire de l'ancienne langue française et de tout ses dialectes du IX au $X V^{e}$ siècle, Paris, 1881-1902, 10 vol., éd. numérique réalisée en 2002 par Classiques Garnier Numérique), ou l'article « parer» du DMF (op. cit.).

15 Roland BARTHES, «Histoire et sociologie du vêtement», art. cit. Pour Barthes, « l'habillement [qui correspond au fait de parole] est faiblement significatif, il exprime plus qu'il ne notifie, le costume [qui correspond au fait de langue] est au contraire fortement signifiant, il constitue une relation intellectuelle, notificatrice, entre le porteur et son groupe» (p.437). Le fait de costume intéresse donc plus Barthes que l'habillement, qui «est constitué par le mode personnel dont un porteur adopte (ou adopte mal) le costume qui lui est proposé par le groupe [et] peut avoir une signification morphologique, psychologique ou circonstancielle, [mais] n'en a pas de sociologique. » (p. 436). 
grammaire vestimentaire ne relève pas seulement d'un lexique, attribuant différentes valeurs à chaque pièce, chaque forme, chaque matière, chaque couleur, mais aussi d'une syntaxe, qui génère, à partir de ces éléments, un discours. Or c'est justement du côté de l'habillement que peut se comprendre la valeur propre de l'ornement, et la conjonction qu'il propose entre une utilité, une signification, et une certaine liberté. La pratique individuelle peut réactiver la valeur symbolique des signes vestimentaires dans une perspective argumentative. Par exemple, les vêtements monastiques peuvent faire l'objet d'une lecture allégorique réintégrant la valeur symbolique des éléments qui avaient d'abord été fonctionnalisés comme signes. Une telle lecture, qui se présente comme un dévoilement du sens, consiste alors simplement en une réactivation des connotations de la pièce d'habillement que ses dénotations intègrent au code social. L'individu peut aussi ajouter des éléments qui n'entreraient pas dans la définition d'une identité : leur signification sera alors plutôt politique, dans un sens large, que sociale. On pourrait ainsi distinguer les valeurs sociales du vêtement, fonctionnalisées et dont la marge de variation serait réduite, des valeurs politiques (et poétiques), qui jouent de cette marge de liberté et lui donnent sens. La dimension esthétique même de l'ornement, alors qu'elle se lie justement à une absence de signification sociale ou politique, est en soi une signification, c'est-à-dire une manipulation du code vestimentaire qui «dit» quelque chose. Elle peut d'ailleurs faire l'objet d'une fonctionnalisation. Gil Bartholeyns a montré comment, au tournant du XIV ${ }^{\mathrm{e}}$ siècle, le développement de la mode comme phénomène social est lié à la recherche, par la noblesse, de nouveaux moyens d'affirmer son identité collective, lorsque le luxe et la richesse n'y suffisent plus. Or l'un des traits qui caractérise la mode est l'anti-fonctionnalité, du point de vue matériel, de vêtements définis par leur rigidité, leur poids, leur démesure. 
Pour pouvoir s'en accommoder, il fallait donc être oisif ${ }^{16}$. La raison sociale récupère ainsi l'ornement et sa gratuité, pour les fonctionnaliser : ils n'ont alors plus seulement un sens circonstanciel, mais une valeur différentielle au sein du code social. C'est sans doute ce qui explique le glissement de sens de parure qui s'observe à la même époque: alors que dans le dictionnaire de Godefroy, seul le sens d'«ornement» est signalé, dans l'article «parure » du DMF, sont répertoriés de nombreux exemple où le terme renvoie à la livrée, l'ensemble des vêtements fournis à la troupe d'un seigneur $^{17}$.

En ce sens, l'ornement s'intègre bien au message communiqué par le vêtement, comme un ajout signifiant. Il n'y aurait donc pas d'éléments plus « gratuits» que d'autres, tous ayant une fonction. Cette unité profonde du vêtement se traduit dans le fait que l'habit, pour le Moyen Âge, englobe l'ensemble de la vêture, des vêtements eux-mêmes à la chaussure, la ceinture ou la coiffure : «mal fait la chappe, qui ne fet le chaperon $»^{18}$. Le Moyen Âge dissocie donc moins que nous ne le faisons les composantes de l'apparence. L'habit, d'ailleurs, avant d'être la tenue, a d'abord été la manière d'être, le maintien habituel d'une personne ou d'un groupe social. Il est donc l'une des composantes du semblant, qui renvoie à l'ensemble des manifestations extérieures de l'être, incluant à la fois les agissements, la physionomie et les vêtements. Le vêtement repose ainsi sur un principe de visibilité, a partie liée avec une dimension éminemment publique, que traduit bien le jeu entre dénotations sociales et connotations politiques.

16 Gil Bartholeyns, «L'enjeu du vêtement au Moyen Âge : de l'anthropologie ordinaire à la raison sociale $\left(\mathrm{XIII}^{\mathrm{e}}-\mathrm{XIV}^{\mathrm{e}}\right.$ siècles "), Le corps et sa parure, Micrologus, 15 (2007), p. 219-273. Pour le développement de la mode, ses causes et ses caractéristiques, voir en particulier p. 233-235.

17 Frédéric Godefroy, Dictionnaire de l'ancienne langue française, op. cit., article "pareüre »; Robert Martin et Bernard Combettes (dir.), Dictionnaire du Moyen Français, op. cit., article « parure ».

${ }^{18}$ Joseph MORAWSKI, Proverbes français..., op. cit., proverbe $\mathrm{n}^{\circ} 1170$. 


\section{«Faire» le moine? La valeur performative du vêtement et l'anthropologie des apparences}

\section{Performativité du vêtement et engagement rituel}

À travers l'ornement, émerge donc l'idée d'une véritable pragmatique du vêtement, au sens linguistique du terme. Le verbe « faire », familier aux proverbes, aurait alors un sens plein, et suggérerait de créditer le vêtement non seulement d'une valeur cognitive, mais d'une véritable valeur performative, illocutoire. Le vêtement ne se contenterait pas de signifier l'être de celui qui le porte, mais contribuerait à le créer. Ainsi, la dimension signifiante du vêtement pourrait être fondée au moins à l'origine sur une dimension performative, assimilable à la portée de certains gestes rituels, qui engagent l'ensemble de la personnalité. Comme pour le langage en effet, certains gestes vestimentaires, institutionnalisés, font plus aisément apparaître la valeur performative du vêtement. Là encore, le choix du moine comme figure proverbiale est caractéristique : la prise d'habit monacal est l'une de ces cérémonies rituelles qui constituent un acte similaire au serment. Par le vêtement, le sujet agit sur lui-même, se transforme, ou plus exactement transforme son ethos social. De ce point de vue, le vêtement marque de véritables rapports d'inclusion dont témoignent, là encore, de nombreuses expressions proverbiales, comme « estre dans la manche de quelqu'un», qui signifie « lui appartenir », ou encore "passer par-dessous la manche", qui signifie «se soumettre à quelqu'un ». Réciproquement, agir « sous le manteau » de quelqu'un, c'est agir en son nom et avec son aval, " tenir quelqu'un sous sa chape », c'est l'avoir en son pouvoir. Enfin, « estre en une chemise », c'est avoir la même volonté, " estre tous d'une livree », subir les mêmes inconvénients. Ce que soulignent toutes ces expressions, c'est que la signification sociale du vêtement ne se comprend pas seulement comme une valeur statique, prise dans un système codé, mais engage très fortement, pour l'homme du 
Moyen Âge, la volonté. Le vêtement n'est pas simplement un signe, mais semble comporter aussi une dimension dynamique et active.

\section{Performativité du vêtement et honorabilité : « en povre robe est li sages foz $»$}

Cette dimension active $\mathrm{du}$ vêtement implique un principe d'honorabilité, qui consiste à paraître ce que l'on est. C'est ce que suggère un proverbe tel que «en povre robe est li sages foz $»^{19}$, où l'inadéquation de son vêtement transforme la nature du sage. La dialectique de la sagesse et de la folie repose ici sur une inversion des signes: une vêture trop humble, déplacée par l'excès d'humilité, révèle en fait une vanité tirée de l'humilité qui anéantit la sagesse. Porter le vêtement adéquat de manière adéquate est donc une marque de mesure, de convenentia, et à ce titre, d'honorabilité : c'est une preuve du sens et de sagesse, qui fait donc le sage au même titre qu'un autre geste. Ce lien entre honneur et paraître se retrouve dans un second proverbe qui engage la figure du fou et la dialectique du comportement sensé ou insensé, «Honte est chapeaus a fol $»^{20}$. Dans ce proverbe, le jugement moral et le vêtement se confondent : témoin de l'honorabilité, le costume en est aussi partie prenante: son adéquation fait partie de l'honorabilité. Cette question de la disconvenance est d'ailleurs le ressort de nombreux récits, et en particulier de fabliaux. Dans Les Braies au cordelier ${ }^{21}$ par exemple, le déshonneur et le ridicule du marchand qui a, par mégarde, revêtu les braies de l'amant de sa femme, un clerc, et trouve à sa ceinture un écritoire plutôt qu'une bourse, n'est pas lié au vêtement en lui-même, mais à sa disconvenance. Il en va de même dans le fabliau de Frère Denise le Cordelier, où Rutebeuf met en scène une

\footnotetext{
${ }^{19}$ Joseph MORAWSKI, Proverbes français..., op. cit., proverbe ${ }^{\circ} 650$.

${ }^{20}$ Ibid., proverbe ${ }^{\circ} 851$.

${ }^{21}$ Willem NoOmen et Nico VAN DEN BoogaARD (éds.), Nouveau Recueil complet des fabliaux, Assen, Van Gorcum, 1983, vol. III, fabliau n ${ }^{\circ}$ 17, p. 211-238
} 
jeune croyante qui, abusée par un cordelier peu scrupuleux, a accepté de se déguiser en moine de cet ordre. Or ce vêtement, en soi respectable, s'avère pour elle moins honorable que ses vêtements de femme, qu'elle reprendra à la fin du fabliau, grâce à l'intervention d'une bourgeoise avisée ${ }^{22}$. L'honorabilité se distingue donc de la respectabilité, qui est plutôt intrinsèque au vêtement, et à l'état social qu'il signifie. Ainsi, tandis que l'honorabilité du vêtement participe d'une éthique, comme le rendent apparent les figures du sage et du fou, sa respectabilité marquerait plutôt ses liens avec l'idéologie, les configurations vestimentaires pouvant, en tant que dispositif signifiant, servir à rendre visibles certaines idées. Le parallèle entre la prise d'habit du moine et l'investiture royale pourrait en être un bon exemple, de même que les caractéristiques communes des vêtements royaux et sacerdotaux, qui tiennent à la question des rapports entre pouvoir temporel et pouvoir spirituel ${ }^{23}$. On a également souvent relevé que la longueur du vêtement avait plutôt, au début du Moyen Âge, une signification sociale, en lien avec l'opposition entre costume de travail et costume de cérémonie, tandis qu'au XIV ${ }^{\mathrm{e}}$ siècle, la longueur du vêtement est associée à l'opposition des $\operatorname{sexes}^{24}$. En ce sens, le fabliau de Rutebeuf marque aussi le rapport de l'apparence avec la notion d'ordre: tous les changements de vêtement ne sont pas légitimes, et l'un des critères qui assurent la validité morale d'un changement de vêtement est son rôle dans le brouillage ou le rétablissement d'un ordre perturbé. C'est ce qui explique qu'un déguisement puisse, contre toute attente puisqu'il contrevient au

\footnotetext{
${ }^{22}$ Rutebeuf, Euvres complètes, Michel Zink (éd.), Paris, LGF, « Le Livre de Poche. Lettres Gothiques », 2001.

${ }^{23}$ Odile Blanc, dans Parades et parures. L'invention du corps de mode à la fin $d u$ Moyen Âge, Paris, Gallimard, "Le temps des images », 1997, donne plusieurs exemples de cette dimension idéologique du vêtement, particulièrement sensible dans les mises en scène des miniatures.

${ }^{24}$ Voir par exemple Jean WIRTH, «Introduction », Le corps et sa parure, Micrologus, 15 (2007), p. 11-41, p. 21 pour les questions de longueur.
} 
principe d'honorabilité, être valorisé, s'il est l'instrument d'un retour à une situation normale.

\section{« Faire semblant » : l'anthropologie des apparences}

Le vêtement semble donc bien doté d'une valeur performative, liée à l'ordre social et aux rituels qui l'engendrent. L'honorabilité est ainsi soustendue par une exigence de visibilité : il est nécessaire de faire semblant en accord avec ses qualités intérieures, de ne pas dissimuler la vertu, mais d'adopter une apparence en accord avec son être. Ainsi, être honorable, pour le Moyen Âge, consiste à paraître ce que l'on est ... mais aussi, par un renversement, à être ce que l'on parait, une fois que l'on a endossé une apparence : endosser une apparence, c'est endosser un être, une nature. $\mathrm{Ce}$ modelage est relativement évident du point de vue du corps. Odile Blanc a ainsi montré comment le jeu des superpositions, des surfaces, des coutures et des fentes pouvait, dans la mode de la fin du Moyen Âge, servir à déplacer les lignes du corps et à le transformer en objet de culture, en y introduisant, par exemple, une forte discontinuitée ${ }^{25}$. L'apparence du corps, ainsi travaillée, est supposée être, selon le principe d'honorabilité, en accord avec l'être. Mais l'influence du vêtement peut aussi se comprendre d'une autre manière, comme le montrent diverses pratiques «magiques" qui prêtent aux vêtements une influence sur le comportement. La version longue du fabliau Les braies au cordelier $^{26}$ présente une pratique qui semble aller dans ce sens. La femme, pour expliquer à son mari comment des braies de cordelier ont pu se retrouver au pied de son lit, explique que ce vêtement lui était destiné : elle l'aurait emprunté à la suite d'un rêve lui prédisant que porter les braies d'un frère mineur lui permettrait de concevoir un enfant. La ruse repose, certes, sur un jeu comique autour des

${ }^{25}$ Odile Blanc, Parades et parures, op. cit., p. 74 sq.

${ }^{26}$ Willem NoOMEN et Nico VAN DEN BOOGAARD (éd.), Nouveau recueil complet des fabliaux, op. cit, vol. III, fabliau n ${ }^{\circ} 17$, p. 211-238. 
supposées valeurs magiques du vêtement, le porteur réel des braies en question pouvant fort bien, mieux que son vêtement, contribuer à cette fécondation. Il n'en reste pas moins que l'argument réussit, suggérant donc, en creux, une «action» du vêtement dont le fabliau s'amuse. Ainsi, si l'engagement personnel dans un ordre social doit bien déterminer l'apparence, le proverbe «l'habit ne fait pas le moine » pourrait aussi viser la dynamique inverse, par laquelle le vêtement servirait à modeler l'être en accord avec un engagement social qui est, dans le même mouvement, un engagement moral $^{27}$. Une série de proverbes, qui ne sont pas liés directement au vêtement, évoque ce jeu de l'être, de l'apparence et de l'action. Les proverbes 155 et 156 de Morawski sont en effet « a tel coutel, tel gayne» et «a tel coutel, tel morsel». Or, d'une façon assez significative, un autre recueil, Toutes les herbes de la Saint-Jean, signale une variante qui inverse le rapport entre les deux propositions, « a tel gaine, tel coustel $»^{28}$. Ces proverbes expriment un rapport de causalité, qu'ils associent à une coïncidence entre le fond et la forme. Le jeu entre " gaine » et « morsel » suggère que les relations entre l'être et le paraître ne sont pas une simple relation binaire, qu'elle soit de coïncidence ou d'opposition, mais un jeu à trois termes entre être, paraître et action. Le vêtement et l'acte sont, au même titre mais avec des modalités différentes, des

${ }^{27}$ De nombreux termes, en ancien français, traduisent cette unité des valeurs sociales et morales. Le couple vilain et courtois en est sans doute l'exemple le plus courant, mais ou peut également penser à des adjectifs comme gentil. Tous ces termes ont en commun de glisser d'une dénomination sociale - est vilain le paysan, courtois l'homme de cour, gentil celui qui appartient à une gens, à une lignée - vers des connotations morales renvoyant à la valeur, et définissant le contenu de celle-ci en fonction de l'idéologie du moment, avec un glissement sensible de la première féodalité, où courtoisie et gentillesse renvoient à la bravoure, à la seconde féodalité, où ils renverront à une valeur mondaine d'élégance, d'art de vivre et d'honnêteté. Gentil, comme gent, peut en outre, appliqué au corps, renvoyer à sa beauté, qui va donc de pair avec la valeur morale et sociale. Gil BARTHOLEYNS donne d'autres exemples de cette association entre bonté et beauté dans son article «L'enjeu du vêtement au Moyen Âge... » (art. cit., p. 236).

${ }^{28}$ Joseph MoRAwski, Proverbes français..., op. cit. ; Giuseppe DI STEFAnO et Rose M. BIDLER, Toutes les herbes de la Saint-Jean, Les locutions en moyen français, Montréal, CERES, « d'hier à demain », 1992, entrée « gaine ». 
manifestations de l'être. La gaine manifeste la nature du couteau, ses vertus latentes, pleinement réalisées dans le «morsel». Mais l'inversion du recueil des Herbes de la Saint-Jean suggère que cette visibilité que la «gaine » procure aux vertus intrinsèques de l'objet ou de l'être repose sur une relation inverse de causalité, où la forme apparente détermine l'être intérieur.

Cette corrélation étroite de l'être et de l'apparence s'inscrit en fait dans le cadre de l'anthropologie chrétienne des interactions entre l'âme et le corps, l'homme intérieur et l'homme extérieur. Le semblant, au Moyen Âge, suppose une corrélation entre l'âme, ou l'être, éternel, et tout ce qui constitue l'apparence : le corps, l'allure, la physionomie du visage, mais aussi le vêtement. S'explique ainsi, par exemple, le fait que la difformité du corps, semblant d'une âme vile, sera fréquemment accompagnée de vêtements déchirés ou bariolés, dont les découpes contrenature traduisent la difformité de l'âme ${ }^{29}$. C'est d'ailleurs un lieu commun chez les moralistes que de dire que les hommes « révèlent leur souillure intérieure par leur tenue $»^{30}$. Entre l'être et son semblant, il n'y a donc pas de solution de continuité ou de contradiction. Mais il résulte de cette interdépendance de l'âme et du semblant que le corps, et à travers lui le vêtement, peuvent aussi agir sur l'âme. Ainsi s'expliquerait que l'habit puisse, ou doive, au sens le plus fort du terme, « faire le moine ». Un terme reflète, au Moyen Âge, cette orientation de l'interaction entre le semblant et les puissances de l'âme : guise. À l'origine, le substantif désigne la manière d'être, la façon. Conformément à la polyvalence du semblant, cette «manière d'être » peut se comprendre à la fois comme une coutume, une

29 Sur ces effets de distorsion des coutures, voir Odile BlanC, Parades et parures, op. cit., p. 50 sq.

30 Orderic Vital, Historia Ecclesiastica, Marjorie ChIBNALl (éd. et trad.), The Ecclesiastical History of Orderic Vitalis, Oxford, Clarendon Press, « Oxford Medieval Texts », 1969, VIII, 190. 
habitude, une manière de se comporter, ou comme une apparence, un aspect: comme dans l'habit, qui est aussi bien «habitude» que «vêtement », agir et paraître se confondent en une même manifestation de l'être. De ce sens, la guise glisse cependant vers celui de «volonté » ou de « désir ». Ce qui est «a la guise » de quelqu'un est ce qui lui plaît, lui convient - la notion de convenance, conçue comme pertinence sociale du point de vue de la sémiotique vestimentaire, se déplace vers une adéquation à l'intention. Quant à la «male guise », c'est la volonté mal orientée. De l'apparence, le substantif glisse vers l'intériorité, sans solution de continuité.

À un «corps miroir de l'âme, modelé par le caractère, l'intentionnalité et les affections subites » répond donc «une intériorité insondable et obscurcie par le corps. [...] les apparences étaient en mesure d'imposer leur lois aux dépens de l'être authentique ». Se crée donc un «phénomène d'identification, où les apparences impliquent l'être [...] porter sérieusement un masque et une peau de bête, c'est s'identifier à elle, en éprouver les qualités physiques. Changer d'apparence modifie l'identité psychologique $»^{31}$. Cette dynamique est particulièrement sensible dans la conception du théâtre médiéval, où le jeu de l'acteur paraissait favoriser,

31 Gil Bartholeyns, «L'enjeu du vêtement au Moyen Âge... », art. cit., p. 225. Un élément serait, du point de vue des interactions entre le corps, le vêtement et l'âme, particulièrement intéressant à analyser: le poil. Par sa polyvalence, il traduit bien l'ambiguïté de cette corrélation. Chez l'homme sauvage, son omniprésence à la place des vêtements est un signe d'animalité, d'ensauvagement, un ensauvagement qui, bien souvent, est lié à une faute d'ordre sexuel, moral. Mais, assez paradoxalement, l'apparence velue se retrouve aussi chez des saints comme Marie l'Égyptienne qui, lorsqu'elle quitte la vie mondaine pour vivre en ermite dans la forêt, finit dépouillée de ses vêtements, et couverte de poils qui se substituent aux draps pour préserver sa pudeur. Leur rôle est donc double. D'un côté, ils manifestent le péché passé de l'Égyptienne, reflètent son âme. Mais, en l'extériorisant, ils permettent aussi de la tenir à distance, de la maîtriser, et donc de la purger. C'est en ce sens qu'ils peuvent se substituer au vêtement comme signe de pudeur et, ce faisant, inverser leur signification traditionnelle. En ce sens, la pratique qui consiste à « fourrer» des vêtements, à faire apparaître, à l'intérieur d'un manteau par exemple, des poils, peut paraître particulièrement ambiguë, et jouer de connotations très différentes. 
par le biais du costume, une identification complète au personnage, comme en témoignent plusieurs anecdotes. Philippe de Vigneulles, dans sa Chronique de Metz, rapporte l'une d'elles : un homme, alors qu'il jouait un diable dans un Mystère, est pris de l'envie de «faire le dyable» avec sa femme, qui, de cette union, conçoit un enfant mi-homme, mi-diable ${ }^{32}$. On remarque que, plus encore que le mouvement qui porte de l'âme vers le corps, qui doit pouvoir impliquer une maîtrise rationnelle de celui-ci, le mouvement qui porte du semblant vers l'homme intérieur est associé à une déformation de l'âme humaine, qui s'éloigne de son statut d'image de Dieu. Ainsi s'explique que l'habit peine à faire le moine: il fera plus aisément le vice que la vertu. Face à ce risque, seul un acte de parole équivalent à l'acte vestimentaire de se costumer peut servir de garantie : on trouve ainsi, dans les actes d'un notaire avignonnais un acte public par lequel un homme devant jouer un diable fait attester que les paroles qu'il prononcera alors seront dites de bouche et non de cœur ${ }^{33}$. Cet acte est intéressant, car, tout en décrivant et en conjurant les risques impliqués par l'interaction entre l'homme intérieur et son semblant, il indique que seule la mise en exergue de l'énonciation et de son contexte singulier permet de légitimer le déguisement en déplaçant l'acte d'énonciation qui consiste à se vêtir sur un autre plan, fictif - laquelle fiction est alors assimilée à l'apparence et au corps, par opposition au «cœur» créant ainsi une dissociation au sein de la personne médiévale.

\footnotetext{
32 Philippe DE Vigneulles, Les Chroniques de la Ville de Metz, Jean-François Huguenin (éd.), Samuel Lamort éditeur, Metz, 1838, p. 473.

${ }^{33}$ Paul PANSIER, "Les débuts du théâtre à Avignon », Annales d'Avignon et du Comtat Venaissin (1919), p. 14, n. 1. Je remercie Simon Gabay de nous avoir présenté ces exemples lors de sa communication au séminaire de Questes et je renvoie, pour une analyse plus approfondie, à son article dans ce bulletin, p. 65-80.
} 
« Contrefaire » le moine ? La maîtrise des apparences et l'arbitraire des signes

Mais la possibilité d'introduire une telle disjonction entre l'être et le paraître n'est pas toujours positive, comme le rappelle notre proverbe, «L'habit ne fait pas le moine, mais la bonne conscience» ou, dans sa version latine, «Habitus non fecit monachus, sed professio regularis $»^{34}$. C'est que la possibilité de disjoindre la guise comme apparence de la guise comme volonté est précisément liée au vêtement. Ce trait est particulièrement apparent à la fin du Moyen Âge, où un auteur comme Christine de Pizan, par exemple, recommande aux dames de savoir dissimuler leurs émotions sous un masque d'impassibilité ${ }^{35}$. Cet idéal rejoint la maîtrise du corps prônée par les religieux. Il s'agit, dans les deux cas, de se préserver de la corruption de l'âme par le corps par un «stoïcisme des apparences» qui «discipliner[ait] les mouvements de l'âme vers le corps et ceux du corps vers l'âme, au moyen de la volonté $»^{36}$. Or l'un des instruments privilégiés de la volonté, pour discipliner le corps, est bien sûr le vêtement, qui permet de se placer dans une position de maîtrise à l'égard de ses apparences. Voilà pourquoi le vêtement, plus que tout autre élément, devrait «faire le moine », en contrariant les influences néfastes de la chair corruptible, voilà pourquoi la sobriété de l'apparence est, pour l'homme vertueux, si essentielle : elle est un moyen du travail sur soi. Le vêtement agit comme une «clôture personnelle » ${ }^{37}$ qui permet de

\footnotetext{
34 Les deux versions sont données par Joseph MoRAWSKI, Proverbes français..., op. cit., proverbe $\mathrm{n}^{\circ} 1053$.

${ }^{35}$ Cet aspect du semblant, et de la nécessité de « faire semblant » en public, lorsque les intentions sont bonnes, a été analysé par Linda Marie RouILLARD. Dans son article "Faux semblant ou faire semblant Christine de Pizan and virtuous artifice », Forum for Modern Language Studies, 46:1 (2010), p. 16-28, elle interprète les recommandations formulées dans le Livre des trois vertus comme une récriture du personnage de Faux Semblant qui réhabilite l'usage du faire semblant.

${ }^{36}$ Gil BARTHOLEYNS, « L'enjeu du vêtement au Moyen Âge... », art. cit., p. 226.

${ }^{37}$ L'expression est de Gil BARThOLEYns, «L'enjeu du vêtement au Moyen Âge... », art. cit., p. 227.
} 
mettre le corps hors jeu. Il devient ainsi à la fois expression de la pureté intérieure, plus fidèle en cela à l'âme que le corps, et moyen du salut qui protège contre les influences de ce même corps. Mais cette position de maîtrise ouvre aussi, béant, l'espace d'un arbitraire des signes, que cristallisent nos proverbes. Car le corps, en même temps que part de l'animal en l'homme, est aussi miroir de vérité. Seul le corps garantit une totale fiabilité de l'apparence comme traduction de l'être intérieur ${ }^{38}$. La « robe », elle, n'est jamais loin de la « lobe», comme le rappelle une rime fréquente dans les œuvres du Moyen Âge ${ }^{39}$. Il n'est alors pas exclu que l'accent mis sur la matérialité du vêtement par certaines versions du proverbe soit tout simplement lié à la dénonciation de dysfonctionnement du processus signifiant. Le choix de parties du vêtement plutôt que d'une dénomination plus abstraite comme «habit» s'inscrirait dans une démarche argumentative implicite, soulignerait, à travers la matérialité du vêtement, son opacité de signe qui voile autant qu'il dévoile.

Enfin, la différence entre le proverbe latin, «habitus non fecit monachus sed professio regularis », et le proverbe français, «l'habit ne fait pas le moine mais la bonne conscience », rappelle que le problème de l'apparence se situe à la croisée de l'arbitraire des signes vestimentaires et de la question éthique de l'intention. Si le «desguisement» peut être parfois bénéfique, parfois hypocrite, c'est bien en fonction de l'intention qui l'anime. La disjonction de l'être et de l'apparence par la « lobe » repose sur une intervention du sujet. Or du point de vue de ce problème éthique,

\footnotetext{
38 Jacqueline CERQUIGLINI-TOULET a exploré cet aspect du rapport au corps dans son article «Syntaxe et syncope: langage du corps et écriture chez Guillaume de Machaut », Langue française, 40 (1978), p. 60-74.

${ }^{39}$ Un auteur comme Rutebeuf, par exemple, qui le premier met un scène un personnage allégorique répondant au nom de Faux Semblant (dans sa Complainte Maistre Guillaume de Saint-Amour, v. 86) en joue de manière récurrente, que ce soit dans ses poésies satiriques ou dans ses poèmes plus personnels, comme les deux Griesches. Voir Rutebeuf, Euvres complètes, op. cit., p. 154-164 pour la Complainte, p. 192-211 pour les Griesches.
} 
les deux proverbes comportent des nuances intéressantes. La "professio regularis » en effet, c'est l'acte performatif par lequel la volonté s'engage, la déclaration publique par laquelle quelqu'un fait profession d'appartenir à un état donné de la société et s'engage à en respecter les règles. Cette version du proverbe semble donc plutôt s'orienter vers une éthique de la shame culture, où « honte est chapeaus a fol », où l'acte public est ce qui engage l'être moral, et où le souci de l'opinion publique, de la fama, permet le contrôle des actes. Ce qui s'oppose à l'habit reste une manifestation extérieure de l'être. Le proverbe crée ainsi, au sein du semblant, une dissociation et une hiérarchie entre plusieurs composantes, dont certaines pourraient exprimer la volonté et la moralité plus fidèlement que d'autres. L'opposition de l'« habit» et de la «bonne conscience » est, elle, plus radicalement, une opposition entre les apparences et l'intériorité, entièrement repliée sur elle-même : on se situerait alors du côté de la guilt culture, où le moi intérieur se fait seul juge de ses actions ${ }^{40}$. Ainsi, ce qui fait le risque du vêtement est le caractère implicite, sous-entendu, de l'acte de langage : en revêtant un vêtement, l'homme est présumé s'engager, mais cet engagement reste un présupposé, dont l'hypocrite peut jouer pour tromper ses semblables. Si « l'habit ne fait pas le moine », c'est à la croisée d'une dynamique de représentation, qui met en exergue l'arbitraire des signes, et de la question éthique de la volonté. N'est-ce pas ce qu'indique Villon qui, dans sa «ballade des menus propos», joue avec son ironie coutumière sur notre proverbe, affirmant tour à tour « Je congnois a la robe l'homme », « Je congnois le moyne a la gonne », et « Je congnois au voile la

\footnotetext{
${ }^{40}$ L'opposition entre shame culture et guilt culture est utilisée par Jean Batany pour interpréter la mise à mort de Malebouche par Faux Semblant dans le Roman de la Rose (Guillaume DE LorRIS et Jean DE Meun, Le Roman de la Rose, Félix LECOY (éd.), Paris, Champion, CFMA, 3 vol., 1065-1970, v. 12003 sq. ; Jean BATANY, Approches du Roman de la Rose, Paris, Bordas, «Bordas Études », 1973, p. 97-111). Il ne faudrait cependant pas lire cette mise à mort comme l'expression d'un rapport de succession entre deux conceptions morales, mais plutôt comme celle d'un conflit, qui se poursuit bien au-delà du XIII ${ }^{\mathrm{e}}$ siècle.
} 
nonne » mais pour toujours conclure, en refrain, « Je congnois tout, fors que moy mesmes $\gg{ }^{41}$ ?

${ }^{41}$ Respectivement v. 2, 10 et 12. François VILlon, Poésies complètes, Claude THIRY (éd.), Paris, Le Livre de Poche, «Lettres Gothiques », 1991. L'ironie joue à la fois dans la personnalisation que subit l'énonciation du proverbe, malicieusement privé de sa négation, et dans le contraste entre le proverbe et le refrain, qui souligne l'inanité de la «connaissance» revendiquée. De fait, sans connaissance de l'âme, comment ses manifestations apparentes, même transparentes, pourraient-elles avoir une quelconque valeur herméneutique? Or de l'âme, Dieu est, pour le Moyen Âge, seul juge et connaisseur. 
